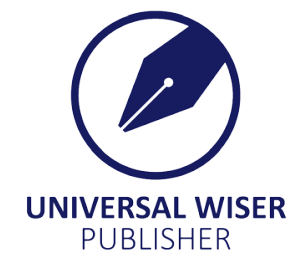

\title{
Optical Properties of Carbon Dots: A Review
}

\author{
Mengli Liu \\ Department of Chemistry and Laboratory of Advanced Materials, Fudan University, Shanghai 200433, P. R. China. \\ E-mail:15226515579@163.com
}

\begin{abstract}
Fluorescent carbon dots (CDs) have attracted increasing attention in recent years owing to their most significant advantages in terms of low toxicity, resistance to photobleaching, small size, easy functionalization, eco-friendly synthesis and diverse imaging capabilities. However, the unclear optical mechanism of CDs greatly limits their further application. Understanding the optical property of CDs is of great significance for controllable development of top-designed CDs with functional purposes. In this review, we firstly summarize that light absorption properties of CDs, and demonstrate the relationship between the absorption spectrum and electron transition of both the core and shell of CDs. Furthermore, we summarize the common fluorescent mechanisms of CDs, including surface state, quantum confinement effect, conjugated structures, self-trapped excitons, edge defects, free zigzag sites, and multi-emissive centers. Finally, we also discuss the phosphorescence properties of CDs. This review gives new insights into how to tune the fluorescence and phosphorescence of the CDs.
\end{abstract}

Key words: Carbon dots, Optical properties, Fluorescent mechanism, Light absorption assignments, Phosphorescence

\section{Introduction}

Fluorescent carbon dots (CDs) consisting of graphene quantum dots (GQDs), carbon nanodots (CNDs) and carbon quantum dots $(\mathrm{CQDs})^{[1,2]}$, as an emerging kind of zero-dimensional carbon nanomaterial have attracted intensive attention from researchers in the fields of bio- and chemo-sensing, cellular imaging, photocatalysis, drug delivery and cancer therapy due to their ultrasmall size, excellent biocompatibility, high loading capacity, tunable fluorescence, and low cost. ${ }^{[3-5]}$ Some review papers about CDs have been published and presented the latest research progress of CDs. Our group ${ }^{[6]}$ summarized $^{6}$ the latest progress of CDs in synthesis, fluorescence and chemiluminescence sensing. Furthermore, we summarized the functionalization strategies of CDs including heteroatom doping and surface modification. ${ }^{[2]}$ Baker's group ${ }^{[7]}$ provided a detailed overview of the current state-of-the-art CDs-incorporated devices and their limitations. Guo's group ${ }^{[8]}$ summarized $^{\text {' }}$ recent research progress of CDs in bioimaging, sensing, catalytic and photoelectronic applications.

Currently, there have been some successful reports of CDs with tunable fluorescence from blue to near-infrared. For example, Lin et al. ${ }^{[9]}$ synthesized the three types of CDs with bright red, green and blue color of fluorescence by varying the carbon sources. Pang et al. ${ }^{[10]}$ prepared a series of CDs with different fluorescence by modifying the reaction conditions. Recently, Huang's group ${ }^{[11]}$ developed a one-pot hydrothermal synthesis route and separated by silica gel column for preparing a fluorescence ladder from blue to near-infrared of CQDs with the absolute quantum yields higher than $70 \%$. Therefore, we can obtain the CDs with different emission by controlling the reaction precursors, temperature, or time.

The tunable fluorescent properties make CDs be a powerful tool for chemo- and biosensing. To make better use of CDs and to tune their optical properties, a full understanding of CDs emission is essential. Unfortunately, a common fluorescent origin of CDs has not yet been proposed even though some breakthroughs have been obtained in recent years. Currently, there are two main opinions for fluorescence origin of CDs: the core state, which is induced by perfect carbon crystals with few defects and modified groups, and the surface state, which is determined by the hybridization of the carbon backbone and connected chemical groups. ${ }^{[5]}$ Very recently, Mandal et al. ${ }^{[12]}$ proposed the whole CDs is fluorescing by rotational anisotropy decay dynamics and resonance energy transfer dynamics. Instead, the fluorescing moiety is either embedded inside the CDs or attached to the surface of the CDs or linked to the CDs through covalent bonds. On the contrary, Ferrante's group ${ }^{[13]}$ revealed the emission properties of CDs, neither based on those cores nor molecular species linked to them but on free molecules, although the presence of carbon core. Therefore, the fluorescent mechanism of CDs is no unified conclusion until now.

Copyright (C2020 Mengli Liu.

DOI: https://doi.org/10.37256/nat.112020124.1-12

This is an open-access article distributed under a CC BY license

(Creative Commons Attribution 4.0 International License)

https://creativecommons.org/licenses/by/4.0/ 
In this review, we summarize the optical properties of CDs, including light absorption assignments, down- and upconversion fluorescence, and phosphorescence, illustrating factors that are important and critical to the guiding significance for controllable synthesis of CDs with specific emission wavelengths. This review will give new insights into how to use different strategies for tuning the optical properties of CDs, understanding the correlation between the absorption spectrum and electron transition of CDs.

\section{Light absorption assignments}

Fluorescent CDs display strong optical absorption in the UV region with a long tail extending into the visible range even to the the NIR region. ${ }^{[14,15]}$ Fig. 1 shows the relationship between the absorption spectrum and the electron transition of both the core and shell of CDs. The shell refers to the surrounded functional groups. The short wavelength bands below $300 \mathrm{~nm}$ (Fig. 1, Band I) correspond to a $\pi \rightarrow \pi *$ transition involving aromatic $\mathrm{sp}^{2}$ carbons (aromatic $\mathrm{C}=\mathrm{C}$ bonds), and the tail starting from $300 \mathrm{~nm}$ to $400 \mathrm{~nm}$ (Fig. 1, Band II) can be assigned to the intrinsic absorption of $\mathrm{n} \rightarrow \pi *$ transition of the $\mathrm{C}=\mathrm{O}$ bond in carbon cores. ${ }^{[16-22]}$ The absorption bands above $400 \mathrm{~nm}$ (Fig. 1, Band III-V) are originated from the surface state transition with electron lone pairs. ${ }^{[18-20]}$ It worth noting that the $\mathrm{n} \rightarrow \pi *$ transition and the broad

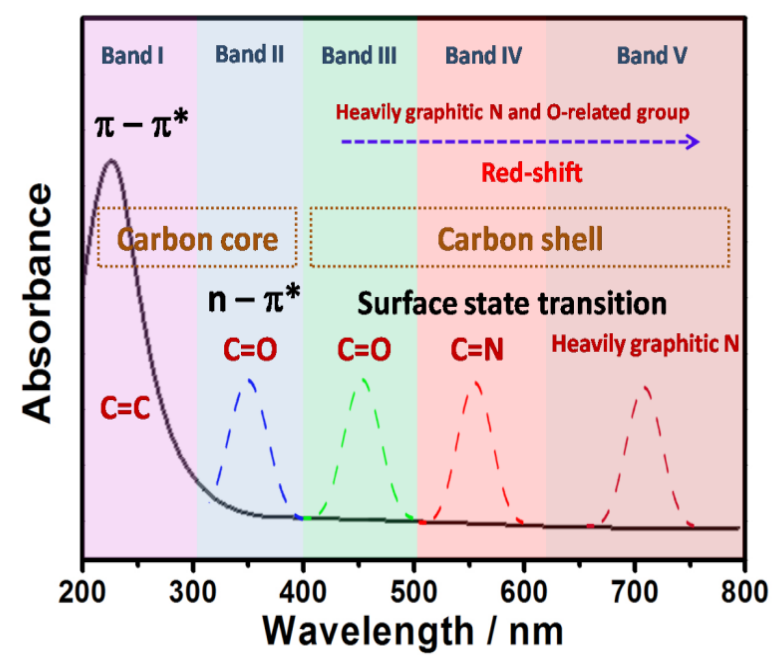

Figure 1. A schematic illustration of the relationship between the absorption spectrum and electron transition of CDs

surface-state absorption bands are usually overlapped, rather than isolated or separated. Therefore, there is no great interruption in the emission tuning, yielding a smooth color gradient as the variation of excitation wavelength. ${ }^{[19]}$ Also note that the low-energy absorption band at about $300 \mathrm{~nm}$ results from $\mathrm{n} \rightarrow \pi *$ and $\pi \rightarrow \pi *$ charge transfer transitions or interlayer charge transfer with a strong $\pi \rightarrow \pi *$ component. $^{[17]}$ In addition, other factors including structural/energetic disorder, medium environment, deprotonation, and excitonic coupling only have a weakly influence on the spectra. ${ }^{[17]}$

The red-shifted UV-vis absorption spectrum from ca. $420 \mathrm{~nm}$ (Fig. 1, Band III) to NIR spectral region (Fig. 1, Band V) is directly attributed to the addition of graphitic nitrogen into the carbon $\mathrm{sp}^{2}$ lattice because the graphitic nitrogen centers can inject excess electron into the unoccupied $\pi^{*}$ orbitals and markedly reduce the HOMO-LUMO gap and the energies of the corresponding optical transitions. ${ }^{[23,24]}$ Meanwhile, O-containing functional group (hydroxyl, carboxyl, and epoxy) on CDs surface can narrow the energy levels, resulting in red-shifted absorption. ${ }^{[10,17,25]}$

\section{Fluorescent properties}

\subsection{Down-conversion fluorescence}

Many efforts have been made to explain the luminescent mechanism of CDs, but there is no unified conclusion until now. Currently, the fluorescent origins of CDs have mainly surface state ${ }^{[10,25-31]}$, quantum confinement effect ${ }^{[9,32,33]}$, conjugated structures ${ }^{[34,35]}$, self-trapped excitons ${ }^{[36,37]}$, edge defects ${ }^{[38-41]}$, free zigzag sites ${ }^{[42-44]}$, multi-emissive centers ${ }^{[45-47]}$. 


\section{$\mathrm{C}-\mathrm{O}-\mathrm{C} / \mathrm{C}-\mathrm{O}$ increase, energy gap reduction}

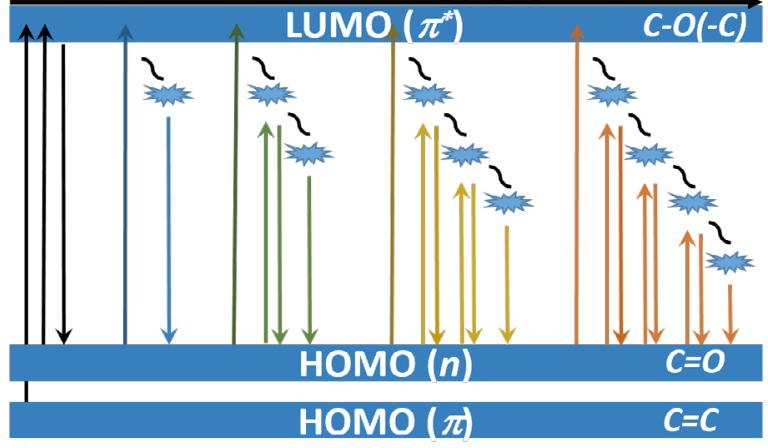

Figure 2. Illustration of the tunable fluorescence emission from CDs containing different O-related surface groups

\subsubsection{Surface state}

The surface state mechanism has been widely studied by researchers. The surface state, including the degree of surface oxidation and surface functional groups, is directly responsible for the fluorescence of CDs. ${ }^{[5,48]}$

The surface oxidation, caused by oxygen-related groups, can create the defect. ${ }^{[25]}$ Different defects can lead to various emission sites on CDs surface, enhancing the CDs fluorescence. ${ }^{[10,25]}$ Mainly, the more surface defects there are, the more excitons (i.e. electrons and holes) can be trapped. ${ }^{[25]}$ Therefore, the radiation from the recombination of trapped excitons leads to the red-shifted emission. The different emissions (blue, green and red fluorescence) of CDs are derived from varied transitions. The blue emission of CDs arises from the radiative recombination of the excited electrons from the $\mathrm{n} \rightarrow \pi *$ transition of $\mathrm{C}=\mathrm{O}$ (Fig. 2) ${ }^{[49]}$ With the increase of O-containing groups (C-OH and C-O-C) on sp ${ }^{2}$-hybridized carbons, numerous epoxide (or hydroxy)-related localized electronic states form below $\pi *$ state, producing numerous new energy levels between $\mathrm{n} \rightarrow \pi *$ gaps (Fig. 2). ${ }^{[49]}$ Therefore, the band gap reduces as oxygen content increases on CDs surface, that is, fluorescence redshift result from the increase in the degree of surface oxidation.

The surface functional groups such as $\mathrm{C}=\mathrm{O}$ and $\mathrm{C}=\mathrm{N}$ groups, also known as the molecular state, can tune the fluorescence of CDs. The O-related groups $(\mathrm{C}=\mathrm{O})$ and N-related groups $(\mathrm{C}=\mathrm{N})$ can introduce two new energy levels $\left(\mathrm{HOMO}-\mathrm{n}_{\mathrm{O}}\right.$ and HOMO $\left.-\mathrm{n}_{\mathrm{N}}\right)$ and further produce new electron transitions (Fig. 3). ${ }^{[20,50]}$ Consequently, the red-shift emission will occur when the electrons in $\mathrm{C}=\mathrm{O}$ or $\mathrm{C}=\mathrm{N}$ groups related defect states return to the HOMO. Furthermore, the white fluorescence is owing to the overlap of diverse light emission induced by electron transitions between the energy levels. $^{[50]}$

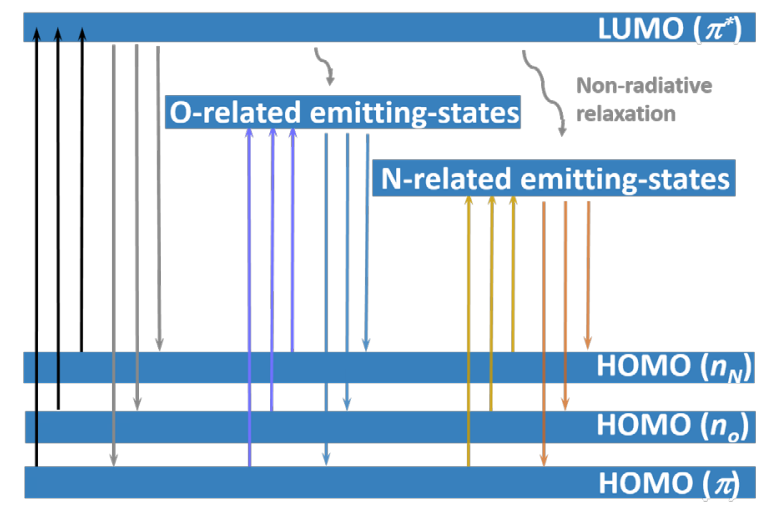

Figure 3. Possible absorption and emission mechanisms for the N-CDs

\subsubsection{Quantum confinement effect}

The GQDs is a zero-dimensional quantum confined system, and its fluorescence has been ascribed to the recombination of electron-hole pairs in quantum-confined GQDs. ${ }^{[42,51,52]}$ In detail, the size of GQDs determines their energy gap, and the gap decreases gradually as the increase of GQDs size. ${ }^{[52,53]}$ In other words, the red-shifted emission of GQDs arises from the increase in size. Li's group ${ }^{[52]}$ demonstrated that the gap decreases gradually as the size of GQDs increases. Therefore, different particle-sized GQDs have different excitation and emission spectra. Furthermore, Choi 
et $a l .{ }^{[33]}$ demonstrated that the anomalous fluorescent behaviors of GQDs in the $5-35 \mathrm{~nm}$ size range are arising from the size-dependent shape and corresponding edge-state variations of GQDs. Lin's group ${ }^{[9]}$ prepared three CDs with red, green and blue fluorescence through a one-pot solvothermal method. The difference in the fluorescent emission for the CDs are attributed to the differences in particle size and nitrogen content. Huang et al. ${ }^{[11]}$ prepared the CDs emitted in full-color from blue to near infrared via a one-pot hydrothermal synthesis route. The fluorescence properties can be coarsely adjusted by tuning the size of the crystalline carbon core due to quantum confinement effects because the sizes of the CDs play a more important role in adjusting the band gap of CDs. With the increase of core size, the extent of $\pi$-electron system gets enlarged, correspondingly, reducing the energy gap between valence band (VB) and conduction band (CB).

\subsubsection{Conjugated structure}

The conjugation system plays an indispensable role in the fluorescence emission of CDs. The large $\mathrm{sp}^{2}$ domain brings about a decrease in the band gap. As a result, the gradually red-shifted fluorescence of CDs is owing to the decreased bandgap energy of CDs as the increasing conjugated structure. ${ }^{[32,35,54,55]}$ Furthermore, Pang's group ${ }^{[10]}$ demonstrated that the energy levels of the electronic states are strict relevant to the intrinsic electronic structure of CDs. The $\pi$-electron domain can strongly couple with surface electronic states created by the surface oxidation, thus influence the entirely electronic structure of the CDs. With the increase in the extent of the $\pi$-electron domain, the energy gap of $\pi \rightarrow \pi *$ transitions decreases and a small energy gap is further caused by the surface states. That is, the red-shifted emission of CDs is derived from the enlarged $\pi$-electron domain or the increase of CDs size.

\subsubsection{Self-trapped excitons}

Self-trapped excitons usually existed both in molecular crystals and in molecular dimers, such as alkali halide crystals, $\mathrm{SiO}_{2}$ and $\mathrm{C}_{60}{ }^{\left[{ }^{[5-58]}\right.}$ In general, a photoexcited exciton can induce a distortion or reorientation in the molecular geometry. The exciton and the distortion can bind together to create a polaron. The exciton becomes localized and stabilized after forming a self-trapped exciton (Fig. 4). ${ }^{[37]}$ The localized bond distortion will produce a strong local potential field, resulting in the self-trapping of the hot carriers.

The emission of CDs is a kind of hot carrier luminescence, which takes place in excited states during the relaxation process, producing a unique feature: the system is not fully degraded and the information (momentum, spin, energy, etc.) of the electronic system induced by the excitation photons is partially/totally conserved. ${ }^{[36]}$ Urban's group $^{[37]}$ demonstrated that the broad Stokes shift in CDs is due to the self-trapping of an exciton in the polycyclic aromatic hydrocarbon (PAH) network. The width and the excitation dependence of the emission come from a selective excitation of PAHs with slightly different energy gaps and from energy transfer between them. Furthermore, Sun's group ${ }^{[36]}$ proved the existence of selftrapped excitons in CDs by using polarization anisotropy spectroscopy and electric-field modulation spectroscopy. The CDs emission originates from radiative recombination of self-trapped excitons, where the mobilization of the carriers is largely impeded due to the existence of a strong local potential field, and thus the relaxation of the hot carriers is considerably suppressed. As compared to itinerant hot free carriers, photo-generated localized exciton excited states in CDs result in a reduced relaxation and degradation process, which explains that the emission tends to shift to longer wavelength/lower energy as time increases.

\subsubsection{Special edge defects}

The fluorescence of CDs can be tuned by defects, which were caused by O-related functional groups at the edge in CDs or originate from two overlapped spectral bands with intrinsic and extrinsic states. The higher the oxygen content on the surface is, the larger the defect is, and the more obvious red shift of the fluorescence will are.

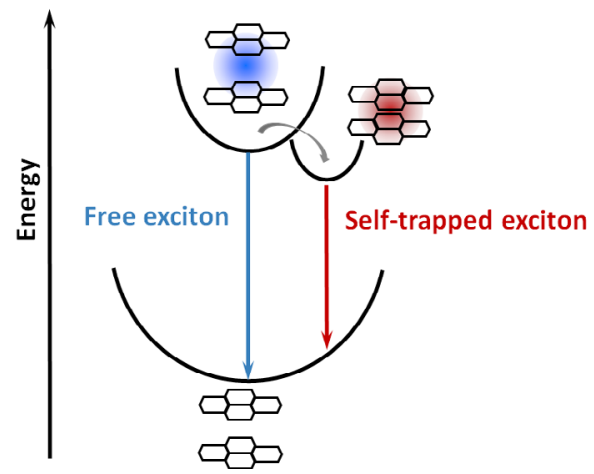

Figure 4. Scheme of the exciton self-trapping process in a pyrene molecule pair: a free exciton (blue spot) may be self-trapped on a molecule pair as a self-trapped exciton (red spot), causing the reduction of energy and mobility 
Seo et al. ${ }^{[39]}$ demonstrated that the blue emission and the robust absorption peak on the higher energy side of GQDs were associated with the formation of the central state in GQDs. However, the green fluorescence of graphene oxide quantum dots (GOQDs), which have more oxygen content than GQDs, was resulted from their abundant oxygenous groups, i.e. defect states. Furthermore, their group ${ }^{[40]}$ proposed that the fluorescence of GQDs is due to the optically excited carriers in GQDs transferred from intrinsic to extrinsic states. The much slower recombination times at short excitation wavelengths than at long excitation wavelengths are because of the carrier transfer, because the carrier transfer increased the relaxation time. In addition, the red-shift behavior of GQDs is related to different numbers of oxygen functional groups of the basal plane and/or the edge. That is, the degree of oxygen content can tune the fluorescence of GQDs.

\subsubsection{Free zigzag sites}

The edge structure of GQDs can significantly affect their electronic structure ${ }^{[44]}$ Therefore, the free zigzag sites on the edge of GQDs can influence the fluorescence of GQDs ${ }^{[59]}$ Wu's group $^{[42]}$ proposed that the fluorescence arises from free zigzag sites with a carbene-like triplet ground state. The great edge effect of GQDs introduced a new UV-vis absorption band at $320 \mathrm{~nm}$. Furthermore, the two electronic transitions of $320 \mathrm{~nm}(3.86 \mathrm{eV})$ and $257 \mathrm{~nm}(4.82 \mathrm{eV})$ obtained in the excitation spectrum of GQDs can be taken as transitions from the $\sigma$ and $\pi$ orbitals (HOMOs) to LUMO. Because the two transitions directly contributed to the blue fluorescence, therefore, the blue emission was the irradiation decay of activated electrons from the LUMO to the HOMO.

\subsubsection{Multi-emissive centers}

The tunable fluorescence of CDs arises from various fluorescent centers, this is the hybridization of multiple small emissive molecules on the emissive carbon cores ${ }^{[47]}$ In general, the blue-green emission mainly results from the graphitic core, while the long-wavelength emission is related to different $\mathrm{C}=\mathrm{O}$ and $\mathrm{C}=\mathrm{N}$ surface functional groups existing in the molecular residues. Besides, the various $\mathrm{N}$ and $\mathrm{O}$ containing conjugated molecular residues can act as different emission centers.

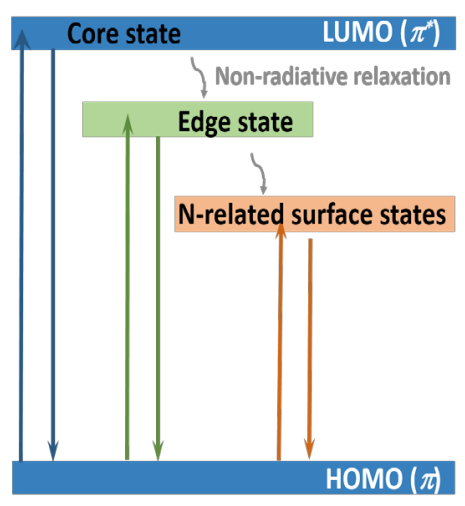

Figure 5. Ternary fluorescent centers-induced fluorescence

The CDs are composed of carbon core and surface state. The carbon core domain is characterized by a $\mathrm{sp}^{2}$ carbon structure, while the surface state contains various functional groups. Lin et al. ${ }^{[45]}$ for the firstly reported the multiemissive states fluorescent behaviors of CDs. The authors demonstrated that the short-wavelength fluorescence (below $400 \mathrm{~nm}$ ) arises from the radiative recombination of electron-hole pairs from the carbon core, whereas the long-wavelength fluorescence (above $400 \mathrm{~nm}$ ) is a result of the radiative relaxation from the excited state to ground state due to the presence of different oxygen-containing groups on CDs surface. Especially, the red-shifted emission initiate from the surface modification with amine groups. The increase of electron density through the addition of lone pair electrons in a nitrogen of amine groups reduces the energy gap, and consequently the emission moves to the longer wavelength.

In addition, Lin et al. ${ }^{[46]}$ proposed that the fluorescence of CDs resulted from ternary fluorescent centers which contained core states, edge states and N-related surface states (Fig. 5). The unique edge states refer to the atoms at the edge of carbon backbone, whereas the surface states are some functional groups adherence on the edge. The core state from $\pi-\pi^{*}$ transitions in CDs contributes to the blue fluorescence, while the edge state from $\mathrm{n}-\pi^{*}$ transitions produces the green fluorescence. The existence of N-related surface states narrowed the energy levels, resulting in a red fluorescent emission center. Therefore, the multi-emissions of CDs arise from various fluorescent centers, corresponding to different parts within one single carbon dot particle. 


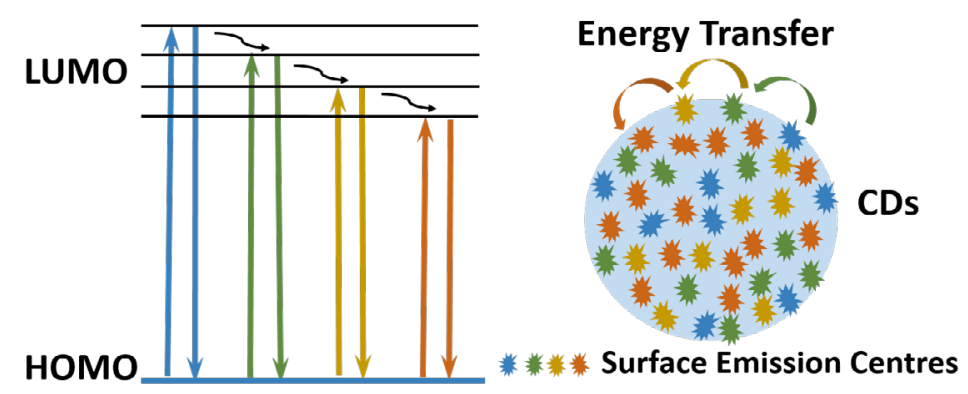

Figure 6. Electron transition diagrams of CDs, and illustration of the integration of multiple surface emission centers on the fluorescent carbon core

Furthermore, the multi-emission behavior of CDs at single particle level were analyzed by a single particle fluorescence imaging technique by Zhang et al. ${ }^{[60]}$, which concretely proved that full-color emissions are single-particle behavior. The authors demonstrated that the functional groups $(-\mathrm{C}=\mathrm{O}$ and $-\mathrm{C}=\mathrm{N})$ can efficiently introduce new energy levels for electron transitions and result in continuously adjustable full-color emissions. Their group ${ }^{[47]}$ further studied the emission behavior of single-particle by total internal reflection fluorescence microscopy. The authors demonstrated that a single carbon dot has multiple emission centers and features of excitation-dependent multi-color emission. Thus, the full-color emission of CDs originated from the hybridization of multiple small fluorescent molecules on the surface and emissive graphitic fragments in the core. Both direct electron transitions of the multi-emission centers separately and energy transfer between different emission-centers all happened in the CDs, resulting in the complicated full-color emission behavior (Fig. 6).

\subsection{Up-conversion fluorescence}

The up-conversion fluorescence is an anti-Stokes emission. The CDs can emit the light with higher energy than the excitation wavelength, that is, the emission wavelength is shorter than the excitation wavelength. ${ }^{[61]}$ Compared with down-conversion fluorescence, the up-conversion fluorescence of CDs has numerous merits for biological applications by the virtue of enhanced tissue penetration depth under NIR and a high signal-to-noise ratio because the long-wavelength fluorescence produced by NIR excitation can avoid the interference of the weak auto-fluorescence background.

The up-conversion fluorescence of CDs is usually considered as a two-photon or multi-photon processes. Sun's group $^{[62]}$ first proposed a two-photon mechanism to explain the up-conversion fluorescence. Liu's group ${ }^{[61]}$ prepared the green-emissive CDs with excellent upconversion fluorescence of high yield of 34\% and a quantum yield (QY) of 16.5\%, which attributed to a multi-photon active process. Rogach's group ${ }^{[15]}$ reported the surface functionalization could endow CDs with NIR absorption band and enhanced NIR emission. The introduction of sulfoxide/carbonyl groups in the outer layers and the edges of the CDs increased surface oxidation, causing a reduction in the LUMO level and producing the redshifted absorption band. Meanwhile, the LUMO levels of the outer layers were lower than those of the inner layers because of the increased surface oxidation, thus producing the NIR absorption band and NIR emission. Moreover, the charge transfers from the inner layers to the outer layers may also contribute to the NIR emission. Given these unique properties, the CDs can simultaneously emit two-photon-induced NIR emission and three-photon-induced red emission when excited at $1400 \mathrm{~nm}$. Kuo's group ${ }^{[63]}$ obtained N-GQDs modified with the polyethylenimine (PEI) and polystyrene sulfonate (PSS) which can act as an active two-photon contrast agent in two-photon bioimaging. The strong electron donation and large $\pi$-conjugated systems, which were arising from the polymers containing $\mathrm{N}$ and $\mathrm{S}$ atoms on the N-GQDs surface, influenced the charge transfer efficiency. The interaction between the N-GQDs and polymers produced oscillating dipoles, therefore, the two-photon emission of polymers modified N-GQDs can cause sharp increases or significant changes. 


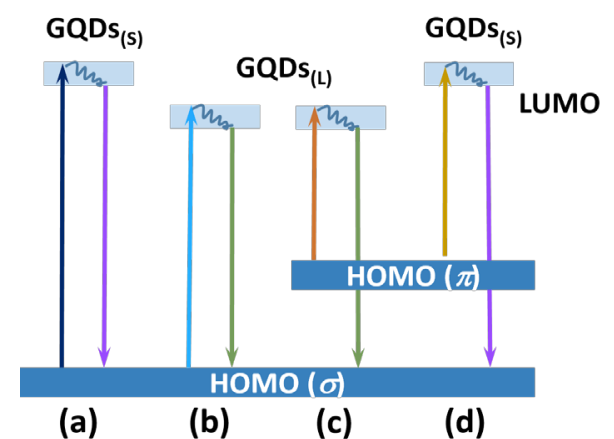
(a)
(b)
(c)
(d)

Figure 7. A schematic illustration of various typical electronic transitions processes of GQDs. Normal fluorescence mechanisms in GQDs for small size (a) and large size (b); Upconverted fluorescence mechanisms in GQDs for large size (c) and small size (d)

However, Li's group ${ }^{[52]}$ argued that the multi-photon excitation is inadequate to account for the up-conversion fluorescence of CDs. The authors prepared GQDs with frequency upconverted emission by a facile hydrazine hydrate reduction of graphene oxide. Given constant energy difference of ca. $1.1 \mathrm{eV}$ between the excitation light and emission light, an energy level structural model for the up-conversion fluorescence properties were constructed as shown in Fig. 7. The up-conversion fluorescence originates from the relaxation of electrons from a higher energy state of the $\pi$ orbital (LUMO) to the $\sigma$ orbital since some electrons would inevitably transit to the LUMO when many low energy photons excite the electrons in the $\pi$ orbital. Although the electrons of the $\sigma$ orbital can also be transitioned, it only can emit conventional down-conversion light.

\subsection{Temperature-dependent emission}

Temperature-dependent fluorescence property has rarely been reported, but it can provide a unique insight into the understanding of the luminescence mechanism of CDs ${ }^{[64-66]}$ Generally speaking, the fluorescent intensity of CDs exhibits a monotonous decrease with increasing temperature, the relaxation dynamics become faster at higher temperatures in the meantime, which could be mainly owing to the occurrence of non-radiative decay processes. ${ }^{[64]}$ The thermal activation of non-radiative trapping may be responsible for non-radiative relaxation. ${ }^{[64]}$ Tang et al. ${ }^{[64]}$ demonstrated that the dual fluorescence bands of CDs arise from core and surface state emission but the energy transfer between core and surface state is ineffective. The very broad fluorescence band is due to the strong electron-electron interactions and weak electronphonon interactions. Liu et al. ${ }^{[65]}$ declared that the thermo-dependent properties of $\mathrm{N}, \mathrm{S}$ co-doped CQDs are attributed to the temperature enhanced population of non-radiative channels of surface (trap/defect) states. More non-radiative channels would be activated at a higher temperature, and more excited electrons returned to the ground state via a non-radiative process, resulting decreased fluorescence intensity.

\subsection{Concentration-dependent emission}

Concentration-dependent fluorescence behavior has been widely concerned by researchers due to its close relationship with surface state emission. A long-wavelength-emitting fluorescent occurs gradually with the increasing of CDs concentration. On the other hand, because of the re-absorption effect at high concentration, the luminescent efficiency decreases as the concentration increasing. ${ }^{[67]}$

Hu et al ${ }^{[68]}$ clarified that the red-shifted emission as the increase of CDs concentration is ascribed to that the radiative recombination of electrons results in the transformation into two or more centers. Furthermore, Lin et al.$^{[4]}$ proposed that the concentration-dependent fluorescent properties are due to the existence of multi-emissive centers in CDs. The muchclosed distance between the emissive centers on particle surface in CDs solution with high concentration can provide a pathway for the non-radiative recombination of charge carriers. The low concentration CDs increases the distance of particle, which weaken the non-radiative relaxation, leading to the enhancement of luminescent efficiency. As for the emission wavelength, the decreased concentration can cause a blue-shift emission owing to the inductive effect and preservation effect.

Nanda et al. ${ }^{[69]}$ elucidated that the tunable fluorescence of the CDs can be controlled by the interparticle distance. At low concentration of CDs, the increase of interpartical distance decreases the interaction among the particles, resulting in the blue-shift with increase of fluorescent intensity due to the radiative process. At higher concentration of CDs, the interparticle distance decreases and the interaction between particles increases, resulting in the red-shift and decrease of fluorescent intensity due to the self-absorption.

\section{5 pH-Dependent emission}


The $\mathrm{pH}$-dependent behavior is also closely relevant with the surface functional groups of the CDs by protonationdeprotonation. The deprotonation may lead to electrostatic doping/charging to CDs and shift the Fermi level. ${ }^{[70]}$

The $\mathrm{pH}$ conditions can dramatically modify the nature of the energy levels and consequently influence the type of electronic transitions involved in CDs. Choudhury et al. ${ }^{[71]}$ demonstrated that red-shifted emission of CDs along with the increased $\mathrm{pH}$ is attributed to the gradual creation of new CDs species in the medium that absorb and emit at longer wavelengths. In addition, the fluorescence intensity of CDs is strong at acid conditions because the strong vibrational coupling of the $\mathrm{OH}$ functional groups, produced by $\mathrm{H}$-bonding effects, can cause the energy level broadening and improve the conformational rigidity of CDs. However, the eliminated H-bonding effect upon deprotonation at basic conditions may lead to the reduced vibrational coupling and more discrete energy levels, which results in the decrease of fluorescence for CDs.

Moreover, Chang et al. ${ }^{[72]}$ prepared $\mathrm{P}$ and $\mathrm{N}$ co-doped CDs with dual fluorescent emissions (R1 and R2) through solvothermal synthesis. Interesting, the $\mathrm{CDs}$ show $\mathrm{pH}$-dependent fluorescence behavior, and $\mathrm{R} 1$ exhibits continuous quenching, while the $\mathrm{R} 2$ opposites with increasing the $\mathrm{pH}$ value. The variant of fluorescence can be easily recognized from the original background with the naked eyes. The authors further clarified that the $\mathrm{N}$ - and P-states are responsible for the effect of $\mathrm{pH}$ variation on $\mathrm{R} 1$ and $\mathrm{R} 2$ emissions, respectively. When $\mathrm{pH}$ value $>7$, the density of the P-state can be enhanced through the combination of P-related groups with $\mathrm{OH}^{-}$radicals, thus promoting $\mathrm{R} 2$ emission. When $\mathrm{pH}$ value $<7$, the protonation of the amine groups would dismiss the unpaired electrons and reduce the electron density in CDs. Not only does this influence the yield of radiative recombination but it also incurs a blue shift of the fluorescent peak.

Lin et al. ${ }^{[45]}$ demonstrated that the $\mathrm{pH}-$ dependent behavior attributed to the carboxylic $(-\mathrm{COOH})$ and amine ($\mathrm{NH}_{2}$ ) functional groups on $\mathrm{CDs}$ surface. The $-\mathrm{COOH}$ and $-\mathrm{NH}_{2}$ have electron-withdrawing and -donating properties, respectively. The $-\mathrm{COOH}$ group at acidic $\mathrm{pH}$ can withdraw the electrons from the conduction band of the carbon-core and hold back electron-hole recombination, causing the fluorescence reduction. On the contrary, the $-\mathrm{COOH}$ group becomes deprotonated $(-\mathrm{COO}-)$ as the $\mathrm{pH}$ increase, thereby reducing the electron-withdrawing capability. As a result, the electronhole recombination becomes feasible, causing the fluorescence enhancement. The $-\mathrm{NH}_{2}$ group cannot enhance the fluorescence under either acidic or basic conditions in comparison to that at $\mathrm{pH}=7$. The fluorescence is not enhanced at basic $\mathrm{pH}$ which is attributed to the electron transfer, whereas the decreased luminescence efficiency at acidic $\mathrm{pH}$ is due to the protonated form $\left(-\mathrm{NH}_{3}{ }^{+}\right)$that may reduce electron-donating efficiency.

However, there are also reports that $\mathrm{pH}$-dependent behavior originates from $\mathrm{pH}$-induced aggregation. Zhang et al. ${ }^{[73]}$ clarified that the red-shifted emission of CDs is due to the formation of CDs aggregates. The CDs can be a well-dispersed solution under low $\mathrm{pH}$ values, however, the $\mathrm{CDs}$ can form the aggregates with increasing the $\mathrm{pH}$ value due to noncovalent molecular interactions, such as hydrogen bonds between the carboxyl groups.

\section{Phosphorescence}

Phosphorescence properties of CDs, especial long-lifetime room-temperature phosphorescence (RTP), have gained wide attention recently. In order to achieve RTP, two prerequisites should primarily be considered: one focuses on enhancing spin-orbit coupling by the introduction of transition metals to promote intersystem crossing efficiency; the other seeks to restrict vibration and rotation to suppress non-radiative transitions. ${ }^{[74,75]}$

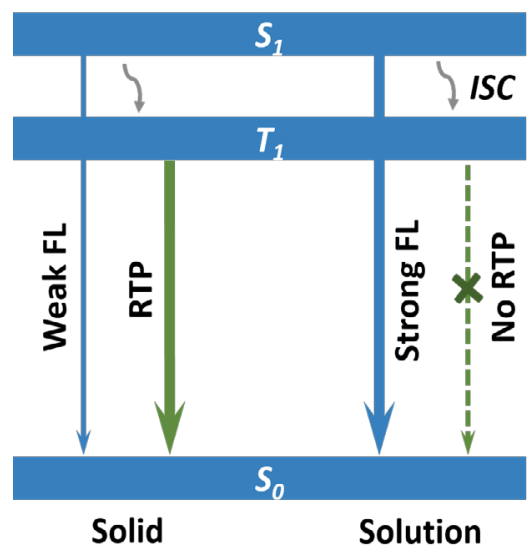

Figure 8. Proposed FL and PL emission processes of the RTP CDs powder and aqueous solution 
CDs with abundant highly cross-linked structures of non-conjugated groups can provide an alternative, novel, and promising approach to achieving the RTP. Yang's group ${ }^{[74]}$ proposed that the polymer CDs can be a new type of RTP materials. The abundant energy levels in the polymer CDs increase the probability of intersystem crossing, and their covalently cross-linked framework structures greatly suppress the non-radiative transitions. Furthermore, the authors demonstrated that the RTP property is directly attributed to a crosslink-enhanced emission effect. Lin's group ${ }^{\text {[75] }}$ further developed that the CDs with long-lifetime RTP (1.46 s). The doping of N and P elements is critical for the ultra-long RTP which is considered to be favored by an $n \rightarrow \pi *$ transition facilitating intersystem crossing (ISC) for effectively populating triplet excitons. In addition, possibilities of the formation of hydrogen bonds in the interior of the CDs may play a significant role in producing RTP. Interesting, the as-prepared CDs emit intense RTP but weak FL in the solid form, the intense RTP showing the effective ISC and stabilization of the excited triplet species, and the weak fluorescence resulting from aggregation-induced quenching; while in solution, they exhibit strong FL but no RTP, a result ascribed to the presence of abundant sub-fluorophores and the disruption of interparticle hydrogen bonds by water in CDs, respectively (Fig. 8).

The RTP in aqueous solution is difficult to achieve because phosphorescence quenching usually occurs in the presence of water owing to the presence of dissolved oxygen as well as the occurrence of solvent-assisted relaxation. ${ }^{\text {[76] }}$ Very recently, Zhou' group ${ }^{[77]}$ utilized bound water to construct hydrogen-bonded networks between CDs and cyanuric acid particles for achieving RTP of CDs in aqueous environments. In the presence of water, the surfaces of the cyanuric acid particles can strongly absorb a layer of highly ordered water molecules (non-freezing bound water). These bound water molecules can construct robust bridge-like hydrogen-bonded networks between the CDs and cyanuric acid particles, which not only effectively rigidifies the $\mathrm{C}=\mathrm{O}$ bonds of $\mathrm{CDs}$ but also greatly enhances the rigidity of the entire system, resulting in the enhancement of phosphorescence.

\section{Conclusions and outlook}

This review is intended to introduce the optical properties of CDs, including light absorption assignments, downand up-conversion fluorescence, and phosphorescence. Many studies about luminescent mechanisms have been published in recent years, but, none of them is convincing and can interpret a large amount of reported data. The CDs prepared by different methods own different size, morphology, or surface state, etc. The fluorescence of CDs is dictated by either the combination or competition between these factors. In addition, the intrinsic structure of CDs is still unclear, and varies with the reaction precursor, temperature and time. Therefore, developing new technologies and strategies to explore the intrinsic structure of CDs is a future research focus. Meanwhile, investigation of the optical properties of CDs includes the different emission centers, excitation dependence, and $\mathrm{pH}$, concentration and solvent sensitivity, all of which are hot research topics. Therefore, more theoretical and experimental methods need to be made to reveal the mystery of CDs fluorescence. In conclusion, the researches on CDs in various fields will continue to increase rapidly in the years to come.

\section{References}

[1] Cayuela A, Soriano ML, Carrillo-Carrion C, et al. Semiconductor and carbon-based fluorescent nanodots: the need for consistency. Chemical Communications. 2016; 52(7): 1311-1326.

[2] Chen BB, Liu ML, Li CM, et al. Fluorescent carbon dots functionalization. Advances in Colloid and Interface Science. 2019; 270: 165-190.

[3] Chen BB, Liu ML, Zhan L, et al. Terbium (III) modified fluorescent carbon dots for highly selective and sensitive ratiometry of stringent. Analytical Chemistry. 2018; 90(6): 4003-4009.

[4] Chen BB, Li RS, Liu ML, et al. Self-exothermic reaction prompted synthesis of single-layered graphene quantum dots at room temperature. Chemical Communications. 2017; 53(36): 4958-4961.

[5] Liu ML, Chen BB, Li CM, et al. Carbon dots: synthesis, formation mechanism, fluorescence origin and sensing applications. Green Chemistry. 2019; 21(3): 449-471.

[6] Liu ML, Chen BB, Li CM, et al. Carbon dots prepared for fluorescence and chemiluminescence sensing. Science China Chemistry. 2019; 62(8): 968-981.

[7] Essner JB, Baker GA. The emerging roles of carbon dots in solar photovoltaics: a critical review. Environmental Science: Nano. 2017; 4(6): 1216-1263.

[8] Du Y, Guo SJ. Chemically doped fluorescent carbon and graphene quantum dots for bioimaging, sensor, catalytic and photoelectronic applications. Nanoscale. 2016; 8(5): 2532-2543.

[9] Jiang K, Sun S, Zhang L, et al. Red, green, and blue luminescence by carbon dots: full-color emission tuning and 
multicolor cellular imaging. Angewandte Chemie International Edition. 2015; 127(18): 5450-5453.

[10] Bao L, Liu C, Zhang ZL, et al. Photoluminescence-tunable carbon nanodots: surface-state energy-gap tuning. Advanced Materials. 2015; 27(10): 1663-1667.

[11] Liu ZX, Zou HY, Wang N, et al. Photoluminescence of carbon quantum dots: coarsely adjusted by quantum confinement effects and finely by surface trap states. Science China Chemistry. 2018; 61(4): 490-496.

[12] Das A, Roy D, De CK, et al. "Where does the fluorescing moiety reside in a carbon dot?"-Investigations based on fluorescence anisotropy decay and resonance energy transfer dynamics. Physical Chemistry Chemical Physics. 2018; 20(4): 2251-2259.

[13] Righetto M, Privitera A, Fortunati I, et al. Spectroscopic insights into carbon dot systems. The Journal of Physical Chemistry Letters. 2017; 8(10): 2236-2242.

[14] Baker SN, Baker GA. Luminescent carbon nanodots: emergent nanolights. Angewandte Chemie International Edition. 2010; 49(38): 6726-6744.

[15] Li D, Jing PT, Sun LH, et al. Near-infrared excitation/emission and multiphoton-induced fluorescence of carbon dots. Advanced Materials. 2018; 30(13): 1705913.

[16] Zhu SJ, Song YB, Zhao XH, et al. The photoluminescence mechanism in carbon dots (graphene quantum dots, carbon nanodots, and polymer dots): current state and future perspective. Nano Research. 2015; 8(2): 355-381.

[17] Sudolská M, Dubecký M, Sarkar S, et al. Nature of absorption bands in oxygen-functionalized graphitic carbon dots. Journal of Physical Chemistry C. 2015; 119(23): 13369-13373.

[18] Zhu JY, Bai X, Bai JL, et al. Emitting color tunable carbon dots by adjusting solvent towards light-emitting devices. Nanotechnology. 2018; 29(8): 085705.

[19] Wang Y, Kalytchuk S, Zhang Y, et al. Thickness-dependent full-color emission tunability in a flexible carbon dot ionogel. The Journal of Physical Chemistry Letters. 2014; 5(8): 1412-1420.

[20] Chen DQ, Wu WW, Yuan YJ, et al. Intense multi-state visible absorption and full-color luminescence of nitrogendoped carbon quantum dots for blue-light-excitable solid-state-lighting. Journal of Materials Chemistry C. 2016; 4(38): 9027-9035.

[21] Liu ML, Chen BB, Yang T, et al. One-pot carbonization synthesis of europium-doped carbon quantum dots for highly selective detection of tetracycline. Methods and Applications in Fluorescence. 2017; 5(1): 015003.

[22] Liu ML, Chen BB, He JH, et al. Anthrax biomarker: an ultrasensitive fluorescent ratiometry of dipicolinic acid by using terbium(III)-modified carbon dots. Talanta. 2019; 191(1): 443-448.

[23] Holá K, Sudolská M, Kalytchuk S, et al. Graphitic nitrogen triggers red fluorescence in carbon dots. ACS Nano. 2017; 11(12): 12402-12410.

[24] Sarkar S, Sudolská M, Dubecký M, et al. Graphitic nitrogen doping in carbon dots causes red-shifted absorption. Journal of Physical Chemistry C. 2016; 120(2): 1303-1308.

[25] Bao L, Zhang ZL, Tian ZQ, et al. Electrochemical tuning of luminescent carbon nanodots: from preparation to luminescence mechanism. Advanced Materials. 2011; 23(48): 5801-5806.

[26] Liu HY, He Zm, Jiang LP, et al. Microwave-assisted synthesis of wavelength-tunable photoluminescent carbon nanodots and their potential applications. ACS Applied Materials \& Interfaces. 2015; 7(8): 4913-4920.

[27] Ding H, Yu SB, Wei JS, et al. Full-color light-emitting carbon dots with a surface-state-controlled luminescence mechanism. ACS Nano. 2016; 10(1): 484-491.

[28] Wang L, Zhu SJ, Wang HY, et al. Common origin of green luminescence in carbon nanodots and graphene quantum dots. ACS Nano. 2014; 8(3): 2541-2547.

[29] Lu Y, Zhang L, Lin HW. The use of a microreactor for rapid screening of the reaction conditions and investigation of the photoluminescence mechanism of carbon dots. Chemistry - A European Journal. 2014; 20(15): 4246-4250.

[30] Song YB, Zhu SJ, Zhang ST, et al. Investigation from chemical structure to photoluminescent mechanism: a type of carbon dots from the pyrolysis of citric acid and an amine. Journal of Materials Chemistry. C 2015; 3(23): $5976-5984$.

[31] Liu ML, Yang L, Li RS, et al. Large-scale simultaneous synthesis of highly photoluminescent green amorphous carbon nanodots and yellow crystalline graphene quantum dots at room temperature. Green Chemistry. 2017; 19(15): 3611-3617.

[32] Yan X, Li BS, Li LS. Colloidal graphene quantum dots with well-defined structures. Accounts of Chemical Research. 2013; 46(10): 2254-2262.

[33] Kim S, Hwang SW, Kim MK, et al. Anomalous behaviors of visible luminescence from graphene quantum dots: interplay between size and shape. ACS Nano. 2012; 6(9): 8203-8208.

[34] Jin SH, Kim DH, Jun GH, et al. Tuning the photoluminescence of graphene quantum dots through the charge transfer effect of functional groups. ACS Nano. 2013; 7(2): 1239-1245.

[35] Qi BP, Hu H, Bao L, et al. An efficient edge-functionalization method to tune the photoluminescence of graphene quantum dots. Nanoscale 2015; 7(14): 5969-5973. 
[36] Xiao L, Wang Y, Huang Y, et al. Self-trapped exciton emission from carbon dots investigated by polarization anisotropy of photoluminescence and photoexcitation. Nanoscale. 2017; 9(34): 12637-12646.

[37] Fu M, Ehrat F, Wang Y, et al. Carbon dots: a unique fluorescent cocktail of polycyclic aromatic hydrocarbons. Nano Letter. 2015; 15(9): 6030-6035.

[38] Cao L, Meziani MJ, Sahu S, et al. Photoluminescence properties of graphene versus other carbon nanomaterials. Accounts of Chemical Research. 2013; 46(1): 171-180.

[39] Liu F, Jang MH, Ha HD, et al. Facile synthetic method for pristine graphene quantum dots and graphene oxide quantum dots: origin of blue and green luminescence. Advanced Materials. 2013; 25(27): 3657-3662.

[40] Song SH, Jang MH, Chung J, et al. Highly efficient light-emitting diode of graphene quantum dots fabricated from graphite intercalation compounds. Advanced Optical Materials. 2015; 2(11): 1016-1023.

[41] Santiago SRM, Lin TN, Yuan CT, et al. Origin of tunable photoluminescence from graphene quantum dots synthesized via pulsed laser ablation. Physical Chemistry Chemical Physics. 2016; 18(32): 22599-22605.

[42] Pan DY, Zhang JC, Li Z, et al. Hydrothermal route for cutting graphene sheets into blue-luminescent graphene quantum dots. Advanced Materials. 2010; 22(6): 734-738.

[43] Chen W, Li FS, Wu CX, et al. Optical properties of fluorescent zigzag graphene quantum dots derived from multiwalled carbon nanotubes. Applied Physics Letters. 2014; 104(6): 063109.

[44] Ritter KA, Lyding JW. The influence of edge structure on the electronic properties of graphene quantum dots and nanoribbons. Nature Materials. 2009; 8(3): 235-242.

[45] Dhenadhayalan N, Lin KC, Suresh R, et al. Unravelling the multiple emissive states in citric-acid-derived carbon dots. Journal of Physical Chemistry C. 2016; 120(2): 1252-1261.

[46] Chen YQ, Lian HZ, Wei Y, et al. Concentration-induced multi-colored emissions in carbon dots: origination from triple fluorescent centers. Nanoscale. 2018; 10(14): 6734-6743

[47] Ju B, Nie H, Liu ZH, et al. Full-colour carbon dots: integration of multiple emission centers into single particles. Nanoscale. 2017; 9(35): 13326-13333.

[48] Xia J, Chen S, Zou GY, et al. Synthesis of highly stable red-emissive carbon polymer dots by modulated polymerization: from the mechanism to application in intracellular $\mathrm{pH}$ imaging. Nanoscale. 2018; 10(47): 2248422492

[49] Hu SL, Trinchi A, Atkin P, et al. Tunable photoluminescence across the entire visible spectrum from carbon dots excited by white light. Angewandte Chemie International Edition. 2015; 54(10): 2970-2974.

[50] Zhang YJ, Yuan RR, He ML, et al. Multicolour nitrogen-doped carbon dots: tunable photoluminescence and sandwich fluorescent glass-based light-emitting diodes. Nanoscale. 2017; 9(45): 17849-17858.

[51] Mueller ML, Yan X, McGuire JA, et al. Triplet states and electronic relaxation in photoexcited graphene quantum dots. Nano Letter. 2010; 10(7): 2679-2682.

[52] Shen JH, Zhu YH, Chen C, et al. Facile preparation and upconversion luminescence of graphene quantum dots. Chemical Communications. 2011; 47(9): 2580-2582.

[53] Li HT, He XD, Kang ZH, et al. Water-soluble fluorescent carbon quantum dots and photocatalyst design. Angewandte Chemie International Edition. 2010; 122(26): 4532-4536

[54] Yan X, Li BS, Cui X, et al. Independent tuning of the band gap and redox potential of graphene quantum dots. Journal of Physical Chemistry Letters. 2011; 2(10): 1119-1124.

[55] Li QQ, Zhang S, Dai LM, et al. Nitrogen-doped colloidal graphene quantum dots and their size-dependent electrocatalytic activity for the oxygen reduction reaction. Journal of the American Chemical Society. 2012; 134(46): 18932-18935.

[56] Matus M, Kuzmany H, Sohmen E. Self-trapped polaron exciton in neutral fullerene $\mathrm{C}_{60}$. Physical Review Letters. 1992; 68(18): 2822-2825.

[57] Kabler MN, Patterson DA. Evidence for a triplet state of the self-trapped exciton in alkali-halide crystals. Physical Review Letters. 1967; 19(19): 652-655.

[58] Itoh C, Tanimura K, Itoh N. Optical studies of self-trapped excitons in $\mathrm{SiO}_{2}$. Journal of Physics C: Solid State Physics. 1988; 21(26): 4693-4702.

[59] Liu ZX, Chen BB, Liu ML, et al. Cu(I)-doped carbon quantum dots with zigzag edge structures for highly efficient catalysis of azide-alkyne cycloadditions. Green Chemistry. 2017; 19(6): 1494-1498.

[60] Nie H, Li MJ, Li QS, et al. Carbon dots with continuously tunable full-color emission and their application in ratiometric $\mathrm{pH}$ sensing. Chemistry of Materials. 2014; 26(10): 3104-3112.

[61] Cui YY, Hu ZB, Zhang CF, et al. Simultaneously enhancing up-conversion fluorescence and red-shifting downconversion luminescence of carbon dots by a simple hydrothermal process. Journal of Materials Chemistry B. 2014; 2(40): 6947-6952.

[62] Cao L, Wang X, Meziani MJ, et al. Carbon dots for multiphoton bioimaging. Journal of the American Chemical 
Society. 2007; 129(37): 11318-11319.

[63] Wu PC, Wang JY, Wang WL, et al. Efficient two-photon luminescence for cellular imaging using biocompatible nitrogen-doped graphene quantum dots conjugated with polymers. Nanoscale. 2018; 10(1): 109-117.

[64] Yu P, Wen XM, Toh YR, et al. Temperature-dependent fluorescence in carbon dots. Journal of Physical Chemistry C. 2012; 116(48): 25552-25557.

[65] Song ZQ, Quan FY, Xu YH, et al. Multifunctional N,S co-doped carbon quantum dots with pH- and thermo-dependent switchable fluorescent properties and highly selective detection of glutathione. Carbon. 2016; 104: 169-178.

[66] Liu WD, Xu SM, Li ZX, et al. Layer-by-layer assembly of carbon dots-based ultrathin films with enhanced quantum yield and temperature sensing performance. Chemistry of Materials. 2016; 28(15): 5426-5431.

[67] Wang C, Hu TT, Wen Zq, et al. Concentration-dependent color tunability of nitrogen-doped carbon dots and their application for iron(III) detection and multicolor bioimaging. Journal of Colloid and Interface Science. 2018; 521(1): 33-41.

[68] Meng X, Chang Q, Xue CR, et al. Full-colour carbon dots: from energy-efficient synthesis to concentration-dependent photoluminescence properties. Chemical Communications. 2017; 53(21): 3074-3077.

[69] Sadhanala HK, Nanda KK. Boron-doped carbon nanoparticles: size-independent color tunability from red to blue and bioimaging applications. Carbon. 2016; 96: 166-173.

[70] Jia XF, Li J, Wang EK. One-pot green synthesis of optically pH-sensitive carbon dots with upconversion luminescence. Nanoscale. 2012; 4(18): 5572-5575.

[71] Dutta Choudhury S, Chethodil JM, Gharat PM, et al. pH-Elicited luminescence functionalities of carbon dots: mechanistic insights. Journal of Physical Chemistry Letters. 2017; 8(7): 1389-1395.

[72] Hu SL, Meng X, Tian F, et al. Dual photoluminescence centers from inorganic-salt-functionalized carbon dots for ratiometric pH sensing. Journal of Materials Chemistry C. 2017; 5(38): 9849-9853.

[73] Wang CX, Xu ZZ, Cheng H, et al. A hydrothermal route to water-stable luminescent carbon dots as nanosensors for $\mathrm{pH}$ and temperature. Carbon. 2015; 82: 87-95.

[74] Tao SY, Lu SY, Geng YJ, et al. Design of metal-free polymer carbon dots: a new class of room-temperature phosphorescent materials. Angewandte Chemie International Edition. 2018; 57(9): 2393-2398.

[75] Jiang K, Wang YH, Gao XL, et al. Facile, quick, and gram-scale synthesis of ultralong room temperature phosphorescent carbon dots by microwave irradiation. Angewandte Chemie International Edition. 2018; 57(21): 6216-6220.

[76] Mukherjee S, Thilagar P. Recent advances in purely organic phosphorescent materials. Chemical Communications. 2015; 51(55): 10988-11003.

[77] Li QJ, Ming Z, Yang MY, et al. Induction of long-lived room temperature phosphorescence of carbon dots by water in hydrogen-bonded matrices. Nature Communication. 2018; 9(1): 734-741. 